Canadian Oncology

Nursing Journal

Revue canadienne

de soins infirmiers

en oncologie

Volume 30, Issue 2 • Spring 2020

elSSN: 2368-8076 


\section{Avoir sa santé en main : le sentiment d'habilitation tel que perçu par les jeunes adultes souffrant d'un cancer avancé}

\section{par Rosalind Garland, Saima Ahmed et Carmen G. Loiselle RÉSUMÉ}

Contexte : Le sentiment d'habilitation sur sa santé (health related empowerment) est un concept fondamental des soins centrés sur la personne. Toutefois, on en sait peu sur la manière dont ce concept s'articule chez les jeunes adultes ayant un diagnostic de cancer avancé.

Objectif: Explorer le sentiment d'habilitation à la lumière des expériences de soins de santé vécues par les jeunes adultes en phase de cancer avancé.

Cadre et participants : Douze jeunes adultes (âgés de 21 à 39 ans) ont été recrutés dans un grand centre de cancérologie de Montréal, au Québec.

Méthodologie : Des entretiens en profondeur durant entre 36 et 90 minutes ont été menés individuellement, enregistrés et retranscrits mot pour mot, puis analysés par thèmes.

Résultats : Tout au long de l'expérience du cancer, les participants ont témoigné du désir soutenu de participer activement à leur traitement et à leurs soins. Quatre thèmes sont ressortis des données décrivant les processus d'attente, de prise en charge de la maladie, de mise en action et de recadrage. Sous-jacents à ces thèmes se trouvent les notions de conscience du corps, les obstacles à surmonter pour obtenir des soins, loptimisation de la santé et la réflexion sur l'héritage qu'on laisse derrière soi.

Conclusions : De façon générale, les participants voulaient demeurer en contrôle de la situation malgré les multiples difficultés inhérentes à un cancer de stade avancé. Si elles sont corroborées par d'autres recherches, ces conclusions pourraient orienter les approches de soins en oncologie afin qu'elles soient véritablement adaptées aux besoins des jeunes adultes.

\section{AUTEURES}

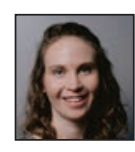

Rosalind Garland, inf. aut., M.Sc.(A), Unité de soins intensifs médico-chirurgicaux, Hôpital général juif, Montréal, Québec

rosalind.garland@mail.mcgill.ca

Saima Ahmed, B.Sc., Division de médecine expérimentale, Université McGill, Montréal, Québec

Saima.ahmed2@mail.mcgill.ca

Carmen G. Loiselle, inf. aut., Ph.D. (auteure-ressource), Affiliation : Université McGill, Département d'oncologie, École de sciences infirmières Ingram, Montréal, Québec; Centre du cancer Segal, Hôpital général juif, Montréal, Québec

Adresse : École de sciences infirmières Ingram et Département d'oncologie, Faculté de médecine, Université McGill

680, rue Sherbrooke Ouest, bureau 1812, Montréal (Québec) H3A $2 M 7$

Tél. : 514-398-4163; Téléc. : 514-398-8455; Courriel : carmen.g.loiselle@mcgill.ca

DOI:10.5737/23688076302103112
Mots clés : cancer avancé; cancer; habilitation; contrôle sur sa santé; oncologie; soutien par les pairs; jeunes adultes

\section{INTRODUCTION}

Chaque année, environ 7600 jeunes adultes canadiens reçoivent un diagnostic de cancer, ce qui en fait la troisième cause de mortalité chez ce groupe (Partenariat canadien contre le cancer, 2017; Statistique Canada, 2019). La recherche montre toutefois que de nombreux besoins des jeunes adultes ne sont pas satisfaits; et le diagnostic tardif, l'agressivité de la maladie et le taux élevé de récidive leur causent bien des difficultés (Anders et al., 2009).

Souvent on catégorise les jeunes entre 18 et 39 ans (Aubin et al., 2011), leur vie étant marquée par plusieurs transitions et défis particuliers comme la recherche d'autonomie et d'indépendance, la poursuite des études et les nouvelles responsabilités professionnelles, de même que les relations amoureuses et les projets de famille (Shaw et al., 2015). On croit que le développement cognitif et social des jeunes adultes, qui diffère de celui des adolescents, est aussi grandement influencé par des facteurs physiques et sociaux (Morgan et al., 2010). En oncologie, les jeunes adultes atteints de cancer rapportent des difficultés : solitude, retrait social, gestion inefficace des symptômes, sentiment de décalage lorsqu'ils participent à des groupes de soutien regroupant des personnes de tous âges, dépendance financière et familiale et, aux stades avancés, transition ardue des soins curatifs aux soins palliatifs et ceux de fin de vie (Borneman et al., 2014; Trevino et al., 2013; Trevino et al., 2014). Lorsque le cancer est avancé, la détresse psychologique liée à l'image corporelle et aux problèmes d'ordre sexuel et reproductif est également présente chez les jeunes adultes (Morgan et al., 2010; Murphy et al.; 2015; Zucchetti et al., 2017). De plus, comme le processus de formation de l'identité n'est pas terminé, le diagnostic de cancer (avancé, chronique ou incurable) a souvent un effet marquant sur l'identité personelle (Kroger et al., 2010; Tindle, 2015; Trevino et al., 2012).

Récemment, on a viserà mieux comprendre l'expérience des jeunes adultes souffrant d'un cancer avancé et la manière d'optimiser leurs efforts d'adaptation psychosociale en faisant appel aux ressources personnelles et aux services en santé (DePauw et al., 2019; Cheung et Zebrack, 2017; Lea et al., 2018; Leuteritz et al., 2017; Quinn et al., 2015; Warner et al., 2018; Zebrack et al., 2013). Le sentiment d'habilitation est bien sûr inhérent à ces processus. Dans le concept d'habilitation, les jeunes adultes sont vus comme des partenaires actifs plutôt que comme des bénéficiaires de soins passifs (Reed et al., 2017).

\section{L'HABILITATION EN MATIÈRE DE SANTÉ}

Au sens large, l'habilitation en matière de santé (HMS) se définit comme le sentiment de pouvoir gérer soi-même son état de santé et de contrôler les questions et les besoins relatifs 
à la santé (Maunsell et al., 2014). L'HMS est étroitement liée à l'engagement et à l'autoreprésentation de ses intérêts, l'engagement étant corrélé à une amélioration des résultats cliniques, à une plus grande satisfaction à l'égard des décisions de traitement et à une réduction du coût des soins de santé (Coulter, 2012; Hibbard et Greene, 2013; Jerofke et al., 2014;). L'HMS est donc considérée comme un élément fondamental des soins centrés sur la personne, de l'activation du patient et de la prise en charge personnelle. Plus précisément, elle est associée à un engagement actif de la personne dans sa vie, à l'apprentissage d'habiletés pour gérer la maladie, à l'adoption d'attitudes constructives et à un bien-être émotionnel positif, de même qu'à la confiance nécessaire pour collaborer et communiquer avec les professionnels de la santé (Maunsell et al., 2014; Osborne et al., 2007).

Dans les cas de cancer, les facteurs multidimensionnels à la base de l'HMS, et de l'habilitation de façon générale, sont associés à une amélioration des résultats de santé (Hibbard et Greene, 2013; Jerofke et al., 2014; Maunsell et al., 2014; van den Berg et al., 2013). En outre, les jeunes adultes souffrant d'un cancer avancé qui entretiennent des relations positives avec les professionnels de la santé se sentent aussi mieux entourés socialement, ont moins d'ennuis de santé et se conforment davantage au traitement (Trevino, Fasciano et Prigerson, 2013). Toutefois, l'un des grands défis de la recherche réside dans l'établissement d'un cadre général d'habilitation des patients en matière de santé afin d'en faire ressortir les principales caractéristiques (Barr et al., 2015; Eskildsen et al., 2017; Maunsell et al., 2014; Zimmerman, 1995). Par conséquent, le moment est venu d'explorer les éléments définitoires de l'HMS en fonctions des différents contextes et groupes de patients.

\section{L'HABILITATION EN MATIÈRE DE SANTÉ ET LES SOINS EN ONCOLOGIE}

Les travaux de Maunsell et collaborateurs (2014) ont observé les facteurs d'habilitation en matière de santé dans les groupes de patients aux prises avec le cancer. Il semble que le soutien social, les attitudes positives et les relations satisfaisantes avec les professionnels de la santé soient fortement liés à une plus grande HMS, de même qu'à l'acquisition de nouvelles habiletés pour gérer le cancer et à une diminution de la détresse émotionnelle (Maunsell et al., 2014). La recherche sur les facteurs influençant l'habilitation en matière de santé suggère également qu'il existe des différences selon le diagnostic et le groupe d'âge (Eskildsen et al., 2017; Mausnell et al., 2014; van den Berg et al., 2013; Zimmerman, 1995).

Certains éléments probants et observations cliniques indiquent aussi que, malgré le stade avancé du cancer, de nombreux patients continuent de participer activement à la prise en charge de leur maladie (Brom et al., 2014; Yennurajalingam et al., 2018). Ces constats s'appliquent aussi aux jeunes adultes, qui veulent demeurer proactifs pour s'adapter aux difficultés causées par le cancer (Trevino et al., 2012). Une étude réalisée par Kaal et collaborateurs (2017) chez les jeunes adultes a mis au jour des associations positives entre l'HMS et la conscience de soi, la capacité de s'adapter à de nouvelles situations, et le soutien social, tandis que les difficultés d'adaptation étaient, quant à elles, corrélées négativement à l'HMS. Pour cerner les interventions prometteuses qui pourraient optimiser l'expérience des soins des jeunes adultes atteints d'un cancer avancé, nous devons d'abord affiner notre compréhension de l'HMS dans ce contexte précis. Par conséquent, nous avons entrepris une recherche qualitative afin de mieux saisir l'opinion et l'expérience des jeunes adultes qui vivent avec un cancer de stade avancé.

\section{MÉTHODOLOGIE}

\section{Questions et devis de l'étude}

La présente étude exploratoire était guidée par les questions de recherche suivantes :

1. Quels sont les facteurs clés qui se dégagent du sentiment d'habilitation en matière de santé tel que le décrivent les jeunes adultes atteints d'un cancer avancé?

2. Quels sont les obstacles et les facteurs favorables à l'habilitation en matière de santé pour ces jeunes adultes?

\section{Participants, milieu et procédures}

Le recrutement s'est déroulé dans un grand centre de cancérologie d'un hôpital de Montréal, au Québec, affilié à l'Université McGill. Dix femmes et deux hommes, âgés entre 21 et 39 ans $(M=33)$ ont accepté de participer à l'étude. Le tableau 1 présente les caractéristiques sociodémographiques des participants. Après approbation par le comité d'éthique, les chercheuses ont procédé à un échantillonnage de commodité dans les organismes communautaires locaux, qui faisaient la publicité de l'étude et demandaient aux éventuels participants s'ils

Tableau 1. Caractéristiques sociodémographiques des participants $(\mathrm{N}=12)$

\begin{tabular}{|l|l|}
\hline Stade & \\
\hline III & 2 \\
\hline IV & 8 \\
\hline Ne sait pas/récidive & 2 \\
\hline Type & \\
\hline Cerveau & 2 \\
\hline Sein & 4 \\
\hline Colorectal & 1 \\
\hline Lymphome hodgkinien & 2 \\
\hline Pancréas & 1 \\
\hline Thyrö̈de & 1 \\
\hline Temps écoulé depuis le diagnostic & \\
\hline Moyenne & 3,2 ans \\
\hline Scolarité & \\
\hline Technique/professionnelle & 5 \\
\hline Baccalauréat ou plus & 7 \\
\hline & \\
\hline
\end{tabular}


acceptaient qu'un agent de recherche communique avec eux pour discuter des exigences de l'étude. Si des jeunes adultes se montraient intéressés, l'agent de recherche en était avisé et prenait contact avec eux, par téléphone ou en personne, pour leur donner les détails de l'étude, répondre à leurs questions et obtenir leur consentement écrit le cas échéant.

Les critères d'inclusion étaient les suivants : 1) être atteint d'un cancer métastatique, chronique ou récurrent de stade III ou IV; 2) pouvoir faire les entrevues en français ou en anglais; 3) avoir entre 18 et 39 ans. Les critères d'exclusion étaient : 1) souffrir de limitations physiques ou cognitives, comme de la fatigue extrême ou des difficultés de communication, empêchant la participation active aux entrevues; ou 2) être en « fin de vie » imminente, c'est-à-dire être considéré comme mourant à cause d'un état de santé qui se détériore rapidement (Jerofke et al., 2014).

\section{Collecte de données}

Après avoir obtenu le consentement éclairé, les auteures principales ont recueilli les données à l'aide d'un guide d'entrevue semi-structurée, d'un enregistreur audio et d'un court questionnaire sociodémographique et médical rempli par les participants.

Le guide d'entrevue a été rédigé à la suite d'une revue exhaustive de la littérature sur l'HMS et les besoins des jeunes adultes atteints de cancer (annexe 1 : Guide d'entrevue pour jeunes adultes atteints de cancer). Quelques questions supplémentaires étaient inspirées du Heath Education Impact Questionnaire mis au point par Osborne et collaborateurs pour étudier l'habilitation en matière de santé (2007). Les questions d'entrevue abordaient des facteurs tels que le soutien social, la collaboration et la prise de décision avec l'équipe médicale, l'acquisition de connaissances sur la maladie, l'expérience des soins, l'autoreprésentation, les interactions avec le système de santé ou les services qui ont été bénéfiques ou problématiques, ainsi que les réseaux de soutien.

La chercheuse rencontrait les participants individuellement, à un moment et un endroit leur convenant à tous deux. Il pouvait s'agir d'une salle privée dans un hôpital, des locaux d'un groupe de soutien communautaire, de l'université ou du domicile du participant. Les entrevues individuelles, d'une durée de 36 à 90 minutes, ont eu lieu entre décembre 2015 et octobre 2016.

\section{Analyse des données}

Les données qualitatives ont fait l'objet d'une analyse thématique selon la méthodologie de Braun et Clarke (2006). D'abord, les enregistrements audio ont été retranscrits mot pour mot, puis les chercheuses ont lu les transcriptions attentivement à plusieurs reprises pour comprendre en détail le contenu général des entrevues. Chacune de leur côté, les auteures ont lu et codé les premières transcriptions, ligne par ligne, pour mettre en évidence le contenu en lien avec les dimensions, obstacles et facteurs favorables de l'HMS. L'une des chercheuses codait les transcriptions, puis discutait du contenu et des thèmes avec l'autre chercheuse pour ensuite en faire une révision. Les données codées étaient classées en catégories cohérentes, puis organisées en thèmes plus généraux. Lorsque de nouveaux thèmes émergeaient, les données déjà codées étaient réexaminées pour en assurer l'exactitude jusqu'à ce que les chercheuses se mettent d'accord et qu'aucun nouveau thème ne se rajoute. Les participants n'avaient pas à commenter les résultats.

Pour améliorer la transparence, les descriptions de l'échantillon et des facteurs contextuels ont aussi été documentées.

\section{RÉSULTATS}

\section{Thèmes centraux}

L'analyse des données a révélé quatre grands thèmes décrivant les liens entre l'HMS et l'autogestion proactive de la maladie chez les jeunes adultes (figure 1).

\section{Thème 1 : Attendez, « c'est mon corps » : Participer} activement aux décisions concernant le soi

1.1 Facteur favorable : Garder le contrôle sur les décisions affectant son corps. De nombreux de participants ont parlé de l'appartenance de leur corps et du fait de devoir vivre avec les effets secondaires des traitements comme raisons de participer activement à la prise de décisions sur leur santé et aux discussions sur les risques et les avantages des traitements proposés. La conscience du corps influençait aussi, dans certains cas, leur décision de continuer le traitement malgré les recommandations contraires des médecins. La conscience de son corps a été clairement nommée par une participante : "C'est mon corps, et si on [lui impose] quoi que ce soit ou que vous prenez une décision, c'est parce que j'aurai dit oui. » (P4)

Certains participants étaient d'avis que même si c'était difficile de contrôler les décisions relatives au traitement, ils devaient tout de même essayer. Ils étaient nombreux à ne pas vouloir être définis par leur maladie et à insister sur le fait que le cancer ne devrait pas altérer outre mesure l'image du corps ou la manière dont les autres perçoivent le désir des patients de prendre part à des activités physiques exigeantes. Les jeunes adultes ont dit qu'ils continuaient à participer à des compétitions sportives, ainsi qu'à des activités en lien avec les études et le travail, à faire des voyages d'aventure, et à vaquer à leurs responsabilités familiales malgré la fatigue chronique, et les effets débilitants et complications des traitements.

Thème 2. Se prendre en charge : Faire tomber les barrières entravant l'accès aux soins et aux services

2.1 Obstacle : Gérer les interactions avec le système de santé. Les jeunes adultes ont décrit les barrières à franchir au moment du dépistage et du diagnostic lorsque les professionnels de la santé ne tenaient pas compte des signes et symptômes qu'ils leur décrivaient. Par exemple, certains médecins de première ligne minimisaient leurs inquiétudes en laissant entendre qu'ils étaient trop jeunes pour s'en faire avec l'idée d'un cancer. Comme le racontait l'une des participantes : «Ils m'ont dit de ne pas m'inquiéter parce que j'étais jeune et que ce n'était probablement rien de grave. » (P7) Les jeunes adultes ont aussi mentionné les interactions négatives, qui leur donnaient un sentiment «d'impuissance » (P9) ou les poussaient à se méfier du système de santé. L’une des participantes a vécu une 
situation où les médecins lui ont menti sur leur expérience avec le traitement du cancer dont elle souffrait :

"J'ai été catégorique sur le fait que je voulais que les médecins qui me traitent... euh... aient de l'expérience et sachent de quoi ils parlaient [...] ils m'ont dit que oui. Mais ce n'était pas le cas [...] et ça n'a pas été facile du tout [...] quand je m'en suis rendu compte après coup [...] » (P4).

Par contre, les jeunes adultes se sentaient plus en possession de leurs moyens lorsque leur voix était entendue, que leurs inquiétudes étaient prises en compte et qu'ils étaient accueillis comme des partenaires actifs dans les discussions concernant leur traitement.

2.2 Obstacle : Composer avec des soins centrés sur le système plutôt que sur la personne. Les participants avaient parfois l'impression que le système de santé lui-même pouvait être un obstacle de taille les empêchant de participer à la prise en charge de leur maladie. Selon eux, le système de santé semble davantage centré sur les besoins des professionnels et des services que sur les patients. Ils ont exprimé très clairement l'importance de faire valoir eux-mêmes leurs besoins malgré leur état de santé précaire (baisses d'énergie, complications, fatigue chronique).

«Je pense que [...] les professionnels de la santé doivent, euh, comprendre que [...] un patient [...] peut parfois avoir trois rendez-vous dans une journée. Essayez de regrouper les rendez-vous dans une même plage horaire. Ne me demandez pas de faire des allers-retours [...] ça exige beaucoup de temps et d'efforts à mon corps et [...] j'essaie de rester en vie. » (P1)

De plus, de l'avis d'autres participants, les protocoles hospitaliers n'étaient pas adaptés à la situation personnelle des patients, leur « voix » étant souvent réduite au silence. Les participants ont aussi mentionné la durée limitée des consultations médicales et les lacunes dans les services, à cause desquelles ils avaient l'impression d'être des numéros plutôt que des partenaires de soins. «On dirait que [...] ils veulent juste en finir, passer au suivant et c'est tout, vous voyez? [...] ce n'est pas un sentiment agréable [...] on a l'impression d'être un numéro et non une personne. » (P7) De plus, les participants ont mentionné les lacunes dans les soins et le manque d'organisation du système, évoquant un «manque de coordination ». (P1) Autre témoignage en ce sens : «Lorsque je suis allé à mon rendez-vous, il m'a dit que je devais refaire les tests parce qu'il avait égaré les résultats. » (P3)

Thème 3 : Agir : Optimiser la santé tout en vivant avec un cancer avancé

3.1 Facteur favorable : Apprendre à manœuvrer dans le monde du cancer. Les participants ont décrit les moyens qu'ils ont pris pour s'y retrouver dans le labyrinthe du cancer tout en préservant leur santé et leur bien-être, par exemple en participant activement à la mise sur pied de leur équipe de professionnels de la santé ou en prenant la décision d'en remplacer les membres au besoin. Voici le raisonnement de l'une des participantes : "Je crois fermement qu'au bout du compte, c'est [...] mon équipe et [que] je choisis mon équipe [...] s'il $y$ a quelqu'un que tu n'aimes pas dans ton équipe, tu as le droit de changer. " (P6) Les participants ont aussi parlé des avantages de prendre eux-mêmes des décisions difficiles au sujet de leur santé et les répercussions sur leur sentiment de contrôle. L'une des participantes explique : «Je suis [...] si fier de moi et je sais que ça prend des efforts [...] du simple fait d'avoir pu changer d'hôpital, vous savez, je suis très fier de moi (rires), j'ai fait quelque chose pour mon bien-être. » (P9) De plus, les jeunes adultes ont décrit les tactiques employées pour composer avec les lacunes ou les délais dans les soins. Certains ont dit avoir

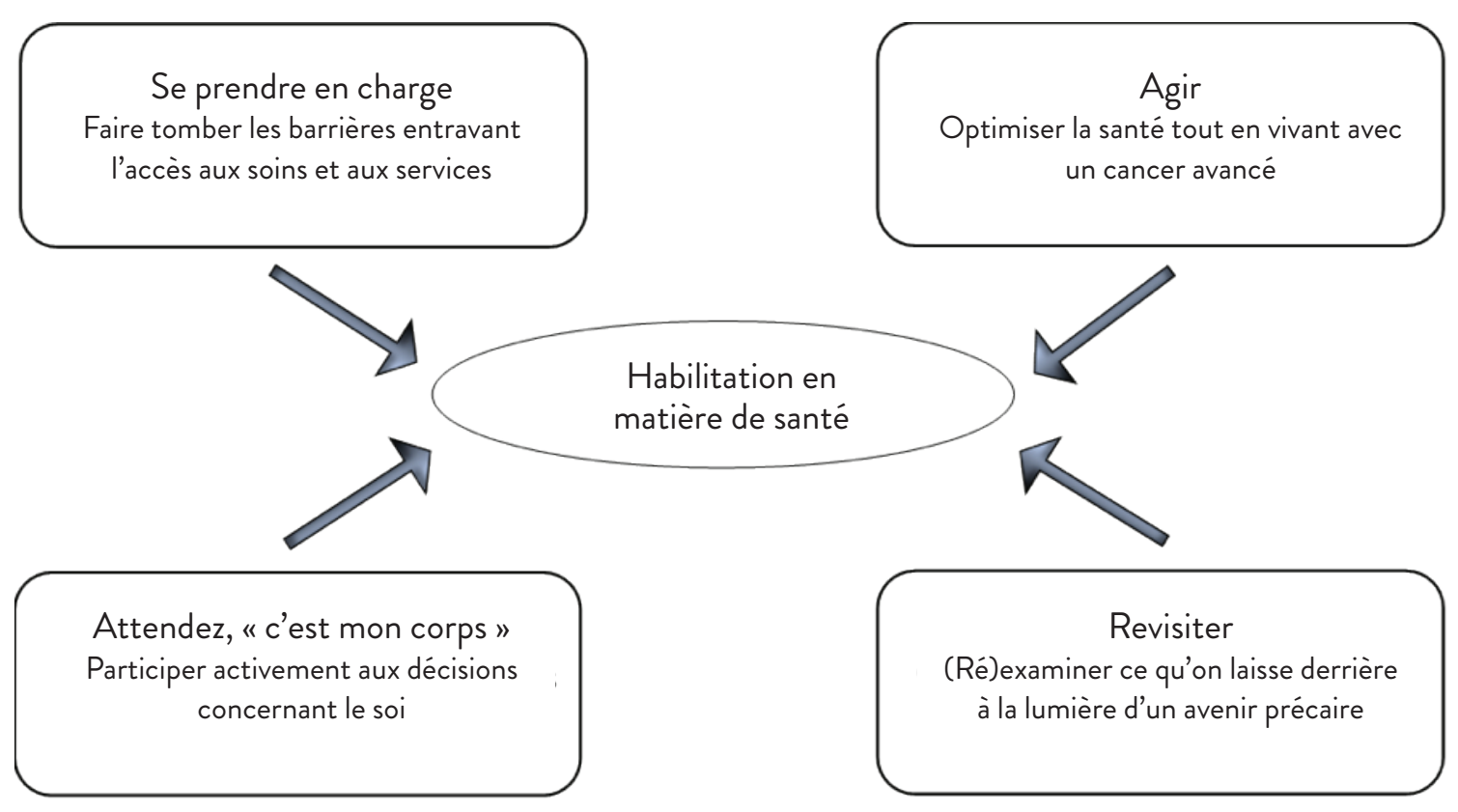

Figure 1. Grands thèmes ressortant de l'analyse thématique qualitative 
étudié les processus structurels du système de santé afin de trouver des stratégies plus efficaces (p. ex. prendre des raccourcis comme l'envoi direct de textos ou d'information par télécopieur à leur médecin) pour réduire les délais et gérer les temps d'attente. Les participants faisaient aussi appel à des gens qu'ils connaissaient, comme des membres de la famille, des pairs, des amis et d'autres patients, pour obtenir l'accès à des ressources et à des traitements complémentaires qu'ils jugeaient bénéfiques.

3.2 Facteur favorable : Chercher de l'information sur le cancer de façon ciblée pour planifier les soins. Tous les participants ont exprimé l'importance d'avoir en main suffisamment d'information pour participer aux décisions concernant leurs soins. Nombre d'entre eux ont dit vouloir disposer de toute l'information pertinente au moins au moment du diagnostic et $\mathrm{du}$ traitement. Dans certains cas, l'information recueillie leur a permis de réclamer de nouvelles options de traitement. En revanche, deux participants (P2, P4) limitaient leur recherche d'information sur le cancer pour ne pas attiser leur anxiété et leurs craintes. Les préférences quant au besoin d'information peuvent aussi changer avec le temps. En voici un exemple :

«Lorsque j'ai reçu le diagnostic de cancer, je je passais mes journées le nez dans un livre ou sur Internet; j'ai aussi essayé d'en discuter avec d'autres pour vraiment comprendre le cancer dans son ensemble [...], mais maintenant, moins j'ai d'information, mieux je me porte. » (P4)

3.3 Obstacle : Composer avec un avenir incertain. Tous les participants ont mentionné l'évolution imprévisible de la maladie et ses répercussions sur leurs projets de vie. Ils ont dû accepter de planifier leurs activités récréatives, leurs vacances et leurs responsabilités à court terme plutôt qu'à long terme. Dans certains cas, les médecins reconnaissaient les difficultés liées à cette grande incertitude face à l'avenir. L'un des participants l'a exprimé ainsi :

"Mon médecin m'a dit... que "personne ne va te blâmer [...] le type de cancer que tu as, c'est comme avoir une guillotine au-dessus de la tête et ne pas savoir quand le couperet va tomber" et, bien que ce soit un commentaire cruel [...] c'est la meilleure représentation visuelle [...] de ce que je ressens. » (P4)

Thème 4 : Revisiter : (Ré)examiner ce qu'on laisse derrière à la lumière d'un avenir précaire

4.1 Facteur favorable : Trouver l'équilibre entre le besoin d'indépendance et de soutien. Les participants tentaient de trouver un juste milieu entre le besoin de demander de l'aide et de conserver leur indépendance. Ils devaient souvent accepter le soutien de la famille et des amis pour accomplir leurs activités quotidiennes, et devaient donc sacrifier l'indépendance dont ils bénéficiaient avant le diagnostic. Cependant, une fois qu'ils étaient à l'aise de demander de l'aide, tous les participants ont éprouvé un grand soulagement. En acceptant du soutien extérieur, plusieurs d'entre eux ont vu qu'ils avaient plus d'énergie à consacrer à d'autres aspects importants de leur vie. Selon un participant :
« Je sais que j'ai de la chance parce que j'ai un bon réseau autour de moi, alors [...] ça me donne de l'énergie pour m'occuper de tout ça. Normalement, quelqu'un qui doit faire tout le reste pourrait ne pas avoir assez d'énergie pour [...] par exemple, faire tous les appels, aller à l'hôpital. » (P12)

4.2 Facteur favorable : Apprécier les relations avec les pairs. Les jeunes adultes ont parlé des nouveaux rôles qu'ils assumaient dans la communauté des patients atteints de cancer. Certains ont parlé de ces relations de soutien avec leurs pairs, qui semblaient mutuellement bénéfiques :

"[Les jeunes adultes] manifestent vraiment [...] le désir de garder le contrôle, et le fait d'approcher [...] la santé [...] et [...] le traitement comme ça, c'est quelque chose qui m'inspire [...] et qui me motive à rester en contrôle [...] et j'espère que je transmets ce sentiment aux autres patients aussi. » (P5)

Un autre a affirmé : « Je suis heureux parce que je me sens utile, malgré la maladie, je peux encore être utile. » (P2) De plus, les jeunes adultes étaient fiers de leur contribution, par exemple en formant de nouveaux groupes de soutien ou en participant à la recherche et à des collectes de fonds.

4.3 Facteur favorable : Choisir son héritage. De nombreux jeunes adultes ont dit que le cancer avait changé l'héritage qu'il voulait laisser derrière eux. L'idée qu'ils s'en faisaient variait, mais il y avait tout de même des points communs. Pour plusieurs, leur vision entre avant et après le diagnostic était très différente. Un participant a dit:

«Je pense que l'héritage que je veux laisser [...] a complètement changé. Hmm, avant, j'imagine que jeétais plus concentré sur ma carrière, je voulais gravir les échelons, travailler fort, bien gagner ma vie [...] et, euh, ça a changé. Maintenant je veux aider les gens, je veux les aider à se faire entendre, je veux les informer, je veux aider tous ceux qui ont un diagnostic semblable au mien. » (P1)

De plus, les jeunes adultes ont dit ne pas vouloir perdre de temps et faire les choses qu'ils ont toujours voulu faire. Ceux qui étaient parents ont souligné, par exemple, que l'enseignement de valeurs morales à leurs enfants était devenu une priorité.

\section{DISCUSSION}

La présente étude offre une fenêtre unique sur le sentiment d'habilitation chez les jeunes adultes qui vivent avec un cancer de stade avancé. Les résultats permettent de mieux comprendre le contexte, les obstacles et les facteurs favorables qui influencent le désir des patients de prendre en charge leur maladie malgré leur état de santé précaire.

Les participants ont signalé être proactifs dans la plupart des aspects de l'expérience du cancer. Ces résultats corroborent ceux des recherches antérieures, qui montrent l'importance, pour les jeunes adultes, de garder le contrôle des décisions touchant leur santé et de participer activement à la prise en charge du cancer (Howk et Wasilewski-Masker, 2011). En pleine transition vers l'indépendance, ils doivent composer avec les soucis d'une maladie de stade avancé et une dépendance périodique envers les autres (Marris et al., 
2011). Chez les jeunes adultes, le côté transformationnel du cancer et la capacité à gérer le fardeau des symptômes constituent des aspects fondamentaux de l'expérience de la maladie (Clark et Fasciano, 2015). Ces besoins, qui s'ajoutent à la volonté de conserver un sentiment de normalité malgré les difficultés, sont également présents chez les patients souffrant de maladies chroniques (D'Agostino, Penney et Zebrack, 2011; Grinyer et Thomas, 2001; Miedema, Hamilton et Easley, 2007). Autres obstacles considérables à l'habilitation : les interactions négatives avec les professionnels de la santé et la rigidité des structures de soins, des points déjà rapportés dans d'autres contextes de soins oncologiques (Maunsell et al., 2014; Mulcahy et al., 2010; van den Berg et al., 2013). Pour les patients qui veulent jouer un rôle plus actif dans les décisions concernant leur maladie, les dynamiques de pouvoir dans les soins de santé se présentent comme un obstacle additionel à surmonter (Bell et al., 2018; Berry et al., 2017).

Les participants ont signalé leur désir d'interagir avec des pairs vivant les mêmes difficultés qu'eux, un souhait également rapporté par d'autres auteurs. L'expertise des pairs est en effet de plus reconnue comme unique et précieuse pour les patients atteints de cancer (Haldar et al., 2018; Hartzler et Pratt, 2011; Power et Hegarty, 2010). Le soutien des pairs et des organisations communautaires est de plus en plus recherché, car il nourrit le sentiment d'habilitation en permettant aux patients de raconter leurs expériences et de bénéficier d'un soutien social, ce qui les aide à trouver l'information nécessaire et à combler leurs besoins émotionnels (Ahmed, Harvey et Amsellem, 2011; Campbell, Phaneuf et Deane, 2004; Lau et al., 2015; Mirrielees et al., 2017; Saxton, Amsellem, Buzaglo et Hollen, 2017). L'importance de l'héritage qu'on laisse derrière, c'est-à-dire la manière dont on veut que les autres se souviennent de nous, fait également partie du sentiment d'habilitation; d'autres chercheurs l'ayant d'ailleurs constaté (Breitbart et al., 2010; Clark et Fasciano, 2015). Les participants à notre étude avaient fait le bilan de leur vie et ressentaient le besoin de laisser une marque positive sur les autres; ces résultats concordent avec les autres recherches sur les jeunes survivants au cancer (Clark et Fasciano, 2015; Lewis et al., 2011; Tindle, 2015).

Les thèmes qui se dégagent de la présente étude sont étroitement liés à l'autoreprésentation de ses intérêts et à l'engagement, reconnus comme des facteurs clés de l'HMS (Coulter, 2012; Hibbard et Greene, 2013; Jerofke et al., 2014; Kaal et al., 2017); l'héritage qu'on laisse derrière soi étant aussi corrélé positivement à l'habilitation chez les jeunes adultes (KeimMalpass, Adelstein et Kavalieratos, 2015).

\section{LIMITES ET CONTRIBUTIONS DE L'ÉTUDE}

La présente étude s'ajoute aux quelques travaux qui ont abordé l'expérience des jeunes adultes atteints d'un cancer. En se penchant sur la manière dont ces jeunes adultes font entendre leur voix dans le système de santé public, ceci correbore l'analyse bibliographique de Drake et Urquhart (2014). Le concept d'HMS décrit dans la présente étude reprend aussi les thèmes relevés par Drake et Urquhart.

Les entretiens en profondeur de la présente étude ont révélé le désir des jeunes adultes de demeurer entièrement engagés dans les soins malgré les difficultés fonctionnelles attribuables au stade avancé de la maladie. Leurs récits personnels détaillés rendent compte de la pertinence de l'HMS pour ce groupe. Les cliniciens doivent encourager les jeunes adultes à être proactifs tout en sachant que leurs besoins et leurs préférences ne cessent d'évoluer. Les infirmières jouent déjà un rôle clé pour défendre et soutenir les patients, mais elles doivent aussi impérativement créer un climat où les voix de ce groupe unique sont entendues par l'équipe multidisciplinaire et invitent les jeunes adultes à participer activement aux décisions concernant leurs soins et leur traitement.

La présente étude comporte toutefois quelques limites qu'il importe de souligner. Les jeunes adultes participants se situaient à la limite supérieure de la tranche d'âge, ce qui peut avoir coloré leur position sur l'habilitation. L'existence d'un programme destiné aux jeunes adultes et la présence d'un organisme bénévole sur les lieux mêmes de l'étude peuvent aussi avoir influencé les récits d'habilitation.

Les recherches à venir devront élucider les variations de l'HMS chez les jeunes adultes atteints de cancer en fonction du sexe, du genre, de la culture, du contexte sociodémographique et de l'éducation.

\section{DÉCLARATION DE CONFLIT D'INTÉRÊTS}

Les auteures déclarent n'avoir aucun conflit d'intérêts.

\section{REMERCIEMENTS}

Nous souhaitons remercier les jeunes adultes qui nous ont généreusement offert de leur temps pour partager leur expérience. Nous voulons aussi remercier "L'espoir, c'est la vie », un organisme communautaire de bénévoles dynamiques, qui nous a aidées à trouver d'éventuels participants, de même qu'Ariella Lang, Ph.D., qui a commenté les premières versions du présent article. L'étude a été financée en partie par des prix remis aux étudiants des cycles supérieurs et décernés à Rosalind Garland par les Instituts de recherche en santé du Canada, la bourse Judith Anne Litvack et la bourse d'étude doctorale Maysie MacSporran. Les recherches de Carmen Loiselle sont financées par la chaire de recherche en oncologie psychosociale Christine et Herschel Victor/L'espoir, c'est la vie de l'Université McGill. 


\section{RÉFÉRENCES}

Ahmed, I., Harvey, A., \& Amsellem, M. (2011). Frankly speaking about cancer: A national model of success for cancer education and patient education. American Public Health Association annual meeting, Washington, D.C., October 29-November 2, 2011.

Anders, C. K., Johnson, R., Litton, J., Phillips, M., \& Bleyer, A. (2009). Breast cancer before age 40 years. Seminars in oncology, 3, 237-249.

Aubin, S., Barr, R., Rogers, P., Schacer, B., Bielack, S.S, Ferrari, A., Manchester, R.A., Morgan, S., Patterson, M., Perlli, A.S., Ribera, J-M., Shaw, P., Thomas, D.M., Treadgold, C. (2011). What should the age range be for AYA oncology? Journal of adolescent and young adult oncology, 1, 3-IO.

Barr, P. J., Scholl, I., Bravo, P., Faber, M. J., Elwyn, G., \& McAllister, M. (2015). Assessment of patient empowerment-A systematic review of measures. PLoS ONE. 10, e0126553. https://doi.org/10.1371/ journal.pone. 0126553 .

Bell, S. K., Roche, S. D., Mueller, A., Dente, E., O'Reilly, K., Sarnoff Lee, B., Sands, K., Talmor, D., \& Brown, S. M. (2018). Speaking up about care concerns in the ICU: Patient and family experiences, attitudes and perceived barriers. BMJ Quality Q Safety, 11, 928-936.

Berry, L. L., Danaher, T. S., Beckham, D., Awdish, R. L. A., \& Mate, K. S. (2017). When patients and their families feel like hostages to health care. Mayo Clinic proceedings, 9, I373-I38I.

Borneman, T., Irish, T., Sidhu, R., Koczywas, M., \& Cristea, M. (2014). Death awareness, feelings of uncertainty, and hope in advanced lung cancer patients: Can they coexist? Int J Palliat Nurs., 20, 27177. https://doi.org/10.12968/ijpn.2014.20.6.271

Braun, V., \& Clarke V. (2006). Using thematic analysis in psychology. Qual Res Psychol., 3, 77-101. https://doi. org/10.1191/1478088706qp063oa

Breitbart, W., Rosenfeld, B., Gibson, C., Pessin, H., Poppito, S., Nelson, C., Tomarken, A., Timm, A. K., Berg, A., Jacobson, C., Sorger, B., Abbey, J., \& Olden, M. (2010). Meaning-centered group psychotherapy for patients with advanced cancer: A pilot randomized controlled trial. Psycho-oncology, 1, 2I-28. https://doi. org/10.1002/pon.1556

Brom, L., Pasman, H. R., Widdershoven, G. A., van der Vorst, M. J., Reijneveld, J. C., Postma, T. J., \& Onwuteaka-Philipsen, B. D. (2014). Patients' preferences for participation in treatment decision-making at the end of life: Qualitative interviews with advanced cancer patients. PloS one, 6, eioo435.

Campbell, H. S., Phaneuf, M. R., \& Deane, K. (2004). Cancer peer support programs - do they work? Patient education and counseling, $1,3-15$.

Canadian Partnership Against Cancer. (2017). Adolescents \& young adults with cancer: A system performance report. Toronto, Ontario: Canadian Partnership Against Cancer.

Cheung, C. K., \& Zebrack, B. (2017). What do adolescents and young adults want from cancer resources? Insights from a Delphi panel of AYA patients. Supportive Care Cancer. 25, 119-126. https://doi. org/10.1007/s00520-016-3396-7

Clark, J. K., \& Fasciano, K. (2015). Young adult palliative care: Challenges and opportunities. Am J Hosp Palliat Care. 32, 101-111. https://doi.org/10.1177/1049909113510394

Coulter, A. (2012). Patient engagement-What works? JACM, 35, 80-89. https://doi:10.1097/JAC.0b013e318249e0fd

D’Agostino, N. M., Penney, A., \& Zebrack, B. (2011). Providing developmentally appropriate psychosocial care to adolescent and young adult cancer survivors. Cancer, 10 Suppl, 2329-2334.
DePauw, S., Rae, C., Schacter, B., Rogers, P., \& Barr, R. D. (2019). Evolution of adolescent and young adult oncology in Canada. Current Oncology, 26, 228-233.

Drake, E. K., \& Urquhart, R. (2019). The experiences of young adults living with metastatic/advanced cancer: A scoping review. Journal of adolescent and young adult oncology. http://doi.org/10.1089/ jayao.2019.0093

Eskildsen, N. B., Joergensen, C. R., Thomsen, T. G., Ross, L., Dietz, S. M., Groenvold, M., \& Johnsen, A. T. (2017). Patient empowerment: A systematic review of questionnaires measuring empowerment in cancer patients. Acta Oncol., 56, 156-165. https://doi.org/10.1080 /0284186x.2016.1267402

Grinyer, A., \& Thomas, C. (2001). Young adults with cancer: The effect of the illness on parents and families. International Journal of palliative nursing, 4, I62-4, I66-70.

Haldar, S., Mishra, S. R., Khelifi, M., Pollack, A. H., \& Pratt, W. (2018). Exploring the design of an inpatient peer support tool: Views of adult patients. AMIA ... Annual Symposium proceedings. AMIA Symposium, I282-I29I.

Hartzler, A., \& Pratt, W. (2011). Managing the personal side of health: How patient expertise differs from the expertise of clinicians. Journal of Medical Internet Research, 3, e62.

Hibbard, J. H., \& Greene, J. (2013). What the evidence shows about patient activation: Better health outcomes and care experiences; fewer data on costs. Health Aff., 32, 207-214. https://doi. org/10.1377/hlthaff.2012.1061

Howk, T., \& Wasilewski-Masker, K. (2011). Palliative care for adolescents and young adults: A pediatric perspective. $J$ Adolesc Young Adult Oncol., 1, 11-12. https://doi.org/10.1089/ jayao.2011.1503

Jerofke, T., Weiss, M., \& Yakusheva, O. (2014). Patient perceptions of patient-empowering nurse behaviours, patient activation and functional health status in postsurgical patients with lifethreatening long-term illnesses. J Adv Nurs., 70, 1310-1322. https:// doi.org/10.1111/jan.12286

Kaal, S. E. J., Husson, O., van Duivenboden, S., Jansen, R., MartenHorst, E., Servaes, P., Prins, J. B., van den Berg, S. W., \& van der Graad, W. T. A. (2017). Empowerment in adolescents and young adults with cancer: Relationship with health-related quality of life. Cancer, 123, 4039-4047. https://doi.org/10.1002/cncr.30827

Keim-Malpass, J., Adelstein, K., \& Kavalieratos, D. (2015). Legacy making through illness blogs: Online spaces for young adults approaching the end-of-life. Journal of Adolescent and Young Adult Oncology, 4, 209-212.

Kroger, J., Martinussen, M., \& Marcia, J. E. (2010). Identity status change during adolescence and young adulthood: A metaanalysis. J Adolesc., 33, 683-698. https://doi.org/10.1016/j. adolescence.2009.11.002

Lau, G. J., Lapointe, J., Dalzell, M. A., Smirnow, N., Ibrahim, M., Beloff, E., Seguin, C., Tremblay, N., Duncan, L., O’Brien, S., \& Loiselle, C. G. (2015, October 4). The breast \& bone health program: A transdisciplinary model of care to proactively address bone health in breast cancer. Canadian Association of Nurses in Oncology annual conference. Toronto, Ontario, October 4-7, 2015.

Lea, S., Martins, A., Morgan, A., Cargill, J., Taylor, R. M., \& Fern, L. A. (2018). Online information and support needs of young people with cancer: Aparticipatory action research. Adolesc Health Med Ther., 9, 121-135. https://doi.org/10.2147/AHMT.S173115

Leuteritz, K., Friedrich, M., Nowe, E., Sender, A., Stöbel-Richter, Y., \& Geue, K. (2017). Life situation and psychosocial care of adolescent and young adult (AYA) cancer patients-Study for protocol of a 
12-month prospective longitudional study. BMC Cancer, 17, 82. https://doi.org/10.1186/s12885-017-3077-z

Lewis, P., Mooney-Somers, J., \& Jordens, C. (2011). "I just want to help people": Complicating young cancer survivors' accounts of "being a better person". Tenth global conference on making sense of health, illness and disease. Oxford.

Marris, S., Morgan, S., \& Stark, D. (2011). 'Listening to Patients': What is the value of age-appropriate care to teenagers and young adults with cancer? Eur J Cancer Care, 20, 145-151. https://doi. org/10.1111/j.1365-2354.2010.01186.x

Maunsell, E., Lauzier, S., Brunet, J., Pelletier, S., Osborne, R. H,, \& Campbell, H. S. (2014). Health-related empowerment in cancer: Validity of scales from the Health Education Impact Questionnaire. Cancer, 120, 3228-3236. https://doi.org/10.1002/ cncr.28847

Miedema, B., Hamilton, R., \& Easley, J. (2007). From "invincibility" to "normalcy": Coping strategies of young adults during the cancer journey. Palliative \& Supportive Care, 1, 4I-49.

Mirrielees, J. A., Breckheimer, K. R., White, T. A., Denure, D. A., Schroeder, M. M., Gaines, M. E., Wilke, L. G., \& Tevaarwerk, A. J. (2017). Breast cancer survivor advocacy at a university hospital: Development of a peer support program with evaluation by patients, advocates, and clinicians. Journal of cancer education: the official journal of the American Association for Cancer Education, 1, 97-9104.

Morgan, S., Davies, S., Palmer, S., \& Plaster, M. (2010). Sex, drugs, and rock ' $n$ ' roll: Caring for adolescents and young adults with cancer. Journal of clinical oncology: official journal of the American Society of Clinical Oncology, 32, 4825-4830.

Mulcahy, C. M., Parry, D. C., \& Glover, T. D. (2010). The "patient patient": The trauma of waiting and the power of resistance for people living with cancer. Qualitative Health Research, 20, 10621075. https://doi.org/10.1177/1049732310369139

Murphy, D., Klosky, J. L., Reed, D. R., Termuhlen, A. M., Shannon, S. V., \& Quinn, G. P. (2015). The importance of assessing priorities of reproductive health concerns among adolescent and young adult patients with cancer. Cancer, 15, 2529-2536.

Osborne, R. H., Elsworth, G. R., \& Whitfield, K. (2007). The Health Education Impact Questionnaire (heiQ): An outcomes and evaluation measure for patient education and self-management interventions for people with chronic conditions. Patient Educ Couns., 66, 192-201. https://doi.org/10.1016/j.pec.2006.12.002

Power, S., \& Hegarty, J. (2010). Facilitated peer support in breast cancer: A pre- and post-program evaluation of women's expectations and experiences of a facilitated peer support program. Cancer Nursing, 2, E9-I6.

Quinn, G., Goncalves, V., Sehovic, I., Bowman, M., \& Reed, D. (2015). Quality of life in adolescent and young adult cancer patients: A systematic review of the literature. Patient Relat Outcome Meas., 6, 19-51. https://doi.org/10.2147/PROM.S51658

Reed, D. R., Oshrine, B., Pratt, C., Fridgen, O., Elstner, C., Wilson, L., Soliman, H., Lee, M. C., McLeod, H. L., Shah, B., Donovan, K. A., Pabbathi, S., Turney, M., Binitie, O., Healy, C., Nieder, M., Shaw, P. H., Galligan, A., Letson, G. D., Stern, M., Quinn, G. P., Davies, S. (2017). Sink or collaborate: How the immersive model has helped address typical adolescent and young adult barriers at a single institution and kept the adolescent and young adult program afloat. Journal of adolescent and young adult oncology, 4, 503-5II.

Saxton, C., Amsellem, M., Buzaglo, J., \& Hollen, H. (2017). Supporting cancer treatment side effect management: Results from a national sample of cancer psychoeducation program attendees. American Public Health Association Annual Meeting, Atlanta, GA, November 4-8, 2017.
Shaw, P. H., Reed, D. R., Yeager, N., Zebrack, B., Castellino, S. M., \& Bleyer, A. (2015). Adolescent and young adult (AYA) oncology in the United States: A specialty in its late adolescence. Journal of pediatric hematology/oncology, 3, I6I-I69.

Statistics Canada. (2019). Leading causes of death, total population, by age group. https://www150.statcan.gc.ca/t1/tbl1/en/ tv. action? pid=1310039401\&pick Members \% 5 B 0\% 5 D = 2.23\&pickMembers\%5B1\%5D=3.1

Tindle, D. A. (2015). Creating meaning: The cancer survivorship experiences of young adults in Australia, England and the United States [PhD]. School of Health and Biomedical Innovation. https:// eprints.qut.edu.au/82216/1/Danielle_Tindle_Thesis.pdf

Trevino, K. M., Abbott, C. H., Fisch, M. J., Friedlander, R. J., Duberstein, P. R., \& Prigerson, H. G. (2014). Patient-oncologist alliance as protection against suicidal ideation in young adults with advanced cancer. Cancer, 120, 2272-2281. https://doi.org/10.1002/ cncr. 28740

Trevino, K. M., Fasciano, K., Block, S., \& Prigerson, H. G., (2013). Correlates of social support in young adults with advanced cancer. Supportive Care Cancer, 21, 421-429. https://doi.org/10.1007/ s00520-012-1536-2

Trevino, K. M., Fasciano, K., \& Prigerson, H. G. (2013). Patientoncologist alliance, psychosocial well-being, and treatment adherence among young adults with advanced cancer. J Clin Oncol., 31, 1683-1689. https://doi.org/10.1200/JCO.2012.46.7993

Trevino, K. M., Maciejewski, P. K., Fasciano, K., Greer, J., Partridge, A., Kacel, E. L., Block, S., \& Prigerson, H. G. (2012). Coping and psychological distress in young adults with advanced cancer. The journal of supportive oncology, 3, I24-I30. https://doi.org/10.1016/j. suponc.2011.08.005

van den Berg, S. W., Van Amstel, F. K. P., Ottevanger, P. B., Gielissen, M. F., \& Prins, J. B., (2013). The cancer empowerment questionnaire: Psychological empowerment in breast cancer survivors. J Psychosoc Oncol., 31, 565-583. https://doi.org/10.1080/ 07347332.2013 .825361

Warner, E. L., Fowler, B., Pannier, S. T., Salmon, S. K., Fair, D., Sparker-Perlman, H., Yancey, J., Randall, R. L., \& Kirchhoff, A. C., (2018). Patient navigation preferences for adolescent and young adult cancer services by distance to treatment location. $J$ Adolesc Young Adult Oncol., 7, 438-444. https://doi.org/10.1089/ jayao.2017.0124

Yennurajalingam, S., Rodrigues, L. F., Shamieh, O. M., Tricou, C., Filbet, M., Naing, K., Ramaswamy, A., Perez-Cruz, P. E., Bautista, M. J. S., Bunge, S., Muckaden, M. A., Fakrooden, S., Sewram, V., Tejedor, A. N., Rao, S. S., Williams, J. L., Liu, D. D., Park, M., Lu, Z., Cantu, H., Hui, D., Reddy, S. K., \& Bruera, E. (2017). Decisional control preferences among patients with advanced cancer: An international multicenter cross-sectional survey. Palliative Medicine, 4, 870-880.

Zebrack, B. J., Block, R., Hayes-Lattin, B., Embry, L., Aguilar, C., Meeske, K. A., Butler, M., \& Cole, S. (2013). Psychosocial service use and unmet need among recently diagnosed adolescent and young adult cancer patients. Cancer, 119, 201-214. https://doi. org $/ 10.1002 /$ cncr.27713

Zimmerman, M. A. (1995). Psychological empowerment: Issues and illustrations. Am J Community Psychol., 23, 581-599. https://doi. org/10.1007/BF02506983

Zucchetti, G., Bellini, S., Bertolotti, M., Bona, F., Biasin, E., Bertorello, N., Tirtei, E., \& Fagioli, F. (2017). Body image discomfort of adolescent and young adult hematologic cancer survivors. $J$ Adolesc Young Adult Oncol., 6, 377-380. https://doi.org/10.1089/ jayao.2016.0067 
Guide d'entrevue pour jeunes adultes atteints de cancer

Merci pour votre participation à cette entrevue.

Le thème de l'entrevue est l'autonomisation lié à la santé, donc nous regardons les aspects qui vous font sentir capable de gérer toutes les choses dont le cancer vous force à faire face et les manières dont vous vous sentez en contrôle ou les difficultés que vous avez à vous sentir en contrôle.

- Pouvez-vous me parler un peu du contexte lorsque vous avez appris que vous aviez le cancer et les soins que vous aviez reçus ?

- (Si ceci n'est pas déjà indiqué dans la réponse précédente) pouvez-vous partagez les lacunes en matière de soins depuis votre diagnostic ?

- Comment décrirez-vous vos relations avec votre équipe de soins (par ex. Infirmiers/infirmières, médecins, physiothérapeute ou autre membre de votre équipe de soins)?

- (Si ceci n'est pas déjà indiqué dans la réponse précédente) Pouvez-vous partager avec moi les interactions positives ou négatives que vous avez eu avec votre équipe de soins depuis votre diagnostic et l'impact que celle-ci ont eu sur votre expérience avec le cancer?

- Pouvez-vous me parler un peu de vos sources de soutien ?

- Que cherchez vous dans vos interactions avec ces individus et jusqu'à date comment vous sentez avec avec ces interactions?

- Qui sont vos plus importantes sources de support (par ex. Ami(e)s, la famille, les groups de soutien, vos collègues de travail, vos professeurs) ?

- En vivant avec une maladie, certaines personnes ressentent que certaines choses leur donnent du pouvoir, et d'autres choses leurs privent de leur pouvoir. Pouvez-vous parler de situations ou d'interactions que vous avez vécu dans le système de la santé où vous vous êtes senti comme si on vous a donner la parole ou que l'on vous a privé de donner votre opinion?

$\circ \quad$ (Si le thème n'est pas élaboré) Pouvez-vous parler de situations où vous vous êtes senti comme si vous puissiez défendre vos propres besoins, ou des situations où vous vous êtes senti contrarie ?

- Pouvez-vous me parler de la manière dont vous gérer les symptômes liés au cancer?

○ Comment avez-vous trouvé ces stratégies ?

- Certaines personnes sentent que leur expérience avec le cancer nuit à une vie avec plénitude, que pensez-vous de ce énoncé ?

- Quand vous pensez à avoir le contrôle sur votre maladie, qu'est-ce-que ceci veut dire pour vous?

○ Pouvez-vous décrire si et comment vous croyez avoir le contrôle sur vos décisions concernant vos traitements?

- Pouvez-vous pensez à une situation dans laquelle vous n'étiez pas en d'accord avec le traitement recommandé par votre équipe traitante et dans cette situation, comment avez-vous géré cette situation? 
- Pouvez-vous me parler d'autres sources de soutien que vous avez recherché par vous-même ou d'autres choses que vous avez recherché pour vous aider?

- (Si ceci n'est pas déjà indiqué dans la réponse précédente) Avez-vous déjà essayé des traitements complémentaires pour lesquel vous n'aviez pas consulté ou discuté avec votre médecin, si oui, pourquoi n'aviez-vous parler avec votre médecin?

- Pouvez-vous vous remettre au moment de votre diagnostic et me parler un peu du changement ou de l'évolution de votre sens de contrôle lié au aspects de votre santé depuis ce moment, celui-ci a t-il changé ?

Merci de donner de votre temps et de partager votre expérience avec nous. Ceci nous informera sur les obstacles auxquels les jeunes peuvent faires face. Nous souhaitons ainsi éclairer la nature des services et des soins offerts aux jeunes adultes en sensibilisant la communauté médicale et le public. 